\title{
D-dimer and C-reactive Protein Blood Levels Over Time Used to Predict Pulmonary Embolism in Two COVID-19 Patients
}

\author{
Yael Becher, Leonid Goldman, Nadav Schacham, Irina Gringauz, Dan Justo \\ Geriatrics and Internal Medicine B Department, Sheba Medical Center, Ramat-Gan, Israel; Aff. to Sackler School of Medicine, Tel-Aviv University, Israel
}

Received: $14 / 05 / 2020$

Accepted: $14 / 05 / 2020$

Published: $20 / 05 / 2020$

\begin{abstract}
How to cite this article: Becher Y, Goldman L, Schacham N, Gringauz I, Justo D. D-dimer and c-reactive protein blood levels over time used to predict pulmonary embolism in two COVID-19 patients. EJCRIM 2020;7: doi:10.12890/2020_001725.
\end{abstract}

Conflicts of Interests: The Authors declare that there are no competing interests.

This article is licensed under a Commons Attribution Non-Commercial 4.0 License

\section{ABSTRACT}

The diagnosis of pulmonary embolism is challenging in symptomatic COVID-19 patients since shortness of breath, chest pain, tachycardia, tachypnoea, fever, oxygen desaturation and high D-dimer blood levels might be features of both diseases. We present two COVID-19 patients in whom pulmonary embolism was suspected (and diagnosed) due to a discrepancy between an increase in D-dimer blood levels and a decrease in C-reactive protein blood levels over time. We believe that an opposite change in the blood levels of both biomarkers over time may be used as a novel method to predict pulmonary embolism in COVID-19 patients.

\section{LEARNING POINTS}

- The diagnosis of pulmonary embolism is challenging in COVID-19 patients since symptoms, signs and high D-dimer blood levels might be similar in both diseases.

- An increase in D-dimer blood levels and a decrease in C-reactive protein blood levels over time may be used as a novel method to predict pulmonary embolism in COVID-19 patients.

\section{KEYWORDS}

COVID-19, C-reactive protein, D-dimer, pulmonary embolism

\section{INTRODUCTION}

Nearly $20 \%$ of COVID-19 patients present severe coagulation abnormalities. Venous thromboembolism (VTE), including pulmonary embolism (PE), has been frequently reported, mainly in critically ill COVID-19 patients and even despite thromboprophylaxis ${ }^{[1]}$. The diagnosis of PE is challenging in symptomatic COVID-19 patients since shortness of breath, chest pain, tachycardia, tachypnoea, fever, oxygen desaturation and high D-dimer blood levels might be features of both diseases ${ }^{[2,3]}$. Herein, we present two COVID-19 patients in whom PE was predicted by a novel method based on measuring D-dimer and C-reactive protein (CRP) blood levels over time.

\section{CASE DESCRIPTIONS}

\section{Case 1}

A 62-year-old man with a history of mild stroke who was taking aspirin for secondary prevention, was admitted due to abdominal discomfort, fatigue, fever, dry cough and shortness of breath. Two weeks previously he had been diagnosed with COVID-19 infection by reverse transcription-polymerase chain reaction (RT-PCR) testing obtained by a nasopharyngeal swab. Physical examination in the emergency room was notable for an oral temperature of $37.3^{\circ} \mathrm{C}$, heart rate of 103 beats/min, respiratory rate of 30 breaths/min, and oxygen saturation 
of 93\% in room air. Laboratory tests showed leucocytosis and neutrophilia, D-dimer blood levels of 1,311 ng/ml, and CRP blood levels of $54 \mathrm{mg} / \mathrm{l}$. Chest and abdomen CT scanning demonstrated infiltrates in the lungs consistent with bilateral pneumonia, and no abdominal pathology, respectively. The patient was treated with oral azithromycin. The patient was not treated with thromboprophylaxis since he was mobile and had no risk factors for VTE other than COVID-19 infection.

Over the next days the patient felt better, dyspnoea almost resolved, oxygen saturation increased to $94-95 \%$ in room air, leucocytosis resolved, and CRP blood levels increased and then decreased to $39 \mathrm{mg} / \mathrm{l}$. However, there was a discrepancy between these signs and laboratory findings and the steady increase in D-dimer blood levels that reached 2,277 ng/ml (Fig. 1). Accordingly, chest CT angiography was performed and demonstrated a left lower lobe PE and lung infarction. Full dose anticoagulation therapy was then started.

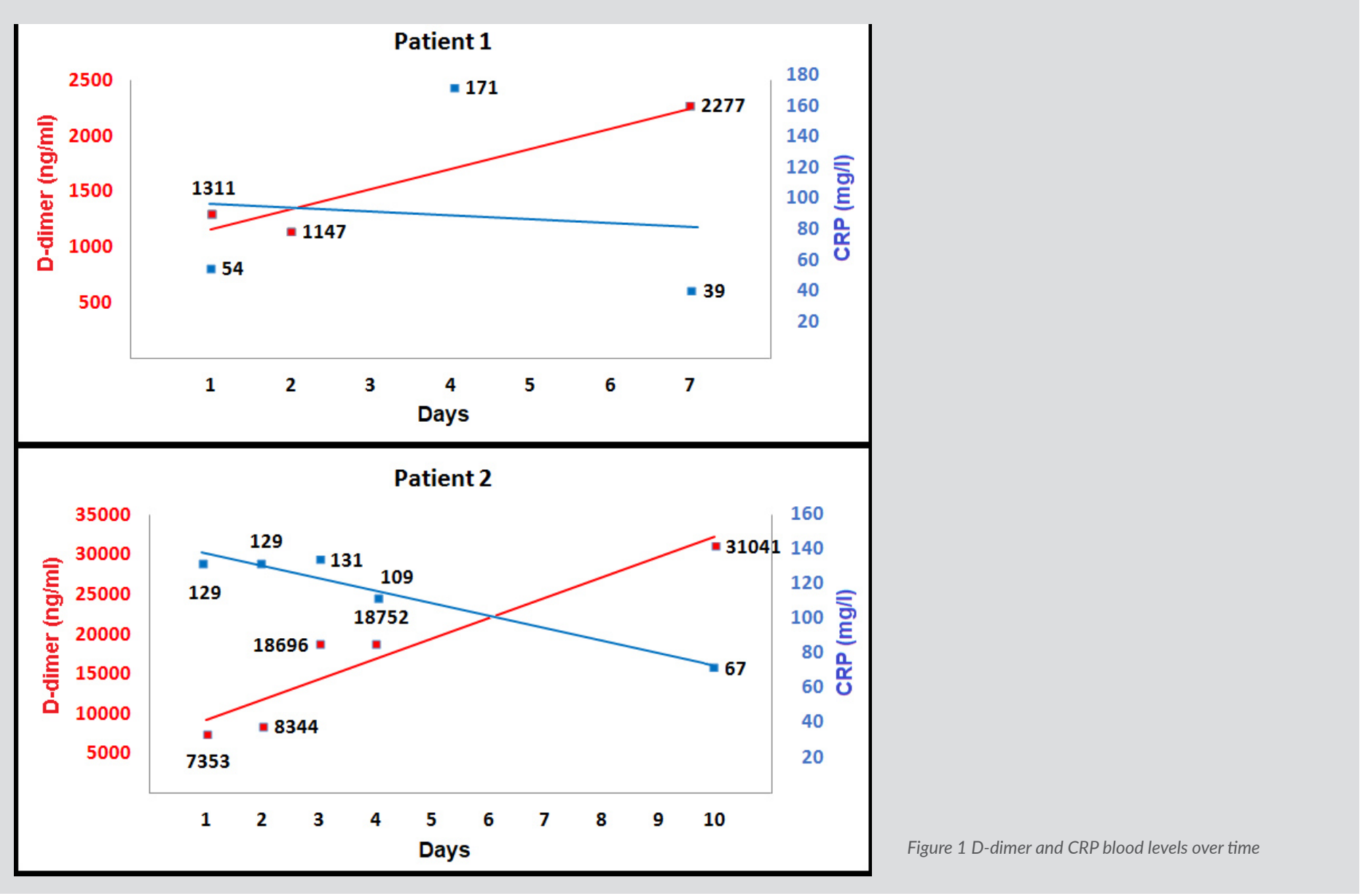

Case 2

A 73-year-old woman with a history of lower limb venous insufficiency, obesity, dyslipidaemia and impaired fasting glucose, who was not taking any medications, was admitted to another hospital due to compressive chest pain. Chest CT angiography demonstrated a proximal ascending aorta dissection. The patient was transferred to our tertiary medical centre and admitted to the cardiac surgery department. Since she came from an endemic area, she was tested for COVID-19 infection by RT-PCR testing of a nasopharyngeal swab, which came back positive. The cardiac surgeons postponed surgery, asked the patient to avoid mobilization, and kept her blood pressure low by administering intravenous labetalol. Physical examination upon arrival at the intensive care unit was notable for an oral temperature of $36.8^{\circ} \mathrm{C}$, heart rate of 72 beats/min, respiratory rate of 24 breaths $/ \mathrm{min}$, blood pressure of 106/55 $\mathrm{mmHg}$, and oxygen saturation of $94 \%$ in room air. Laboratory tests showed no leucocytosis, D-dimer blood levels of 7,353 ng/ml, and CRP blood levels of $129 \mathrm{mg} / \mathrm{l}$. Another chest CT angiogram showed no change in the aortic dissection. The patient was not treated with thromboprophylaxis because of the high risk of aortic rupture and bleeding, but a pneumatic compression device on both legs was used in order to prevent deep vein thrombosis.

Over the next days the patient was asymptomatic, oxygen saturation was normal most of the time, CRP blood levels decreased to 67 
$\mathrm{mg} / \mathrm{l}$, and four RT-PCR tests for COVID-19 infection on nasopharyngeal swabs came back negative. However, there was a discrepancy between these signs and laboratory findings and the steady increase in D-dimer blood levels that reached 3,1041 ng/ml (Fig. 1). A third CT angiogram showed no change in the aortic dissection, but a new bilateral PE was demonstrated (Fig. 2). Thereafter, the patient underwent aortic dissection repair and thrombectomy.

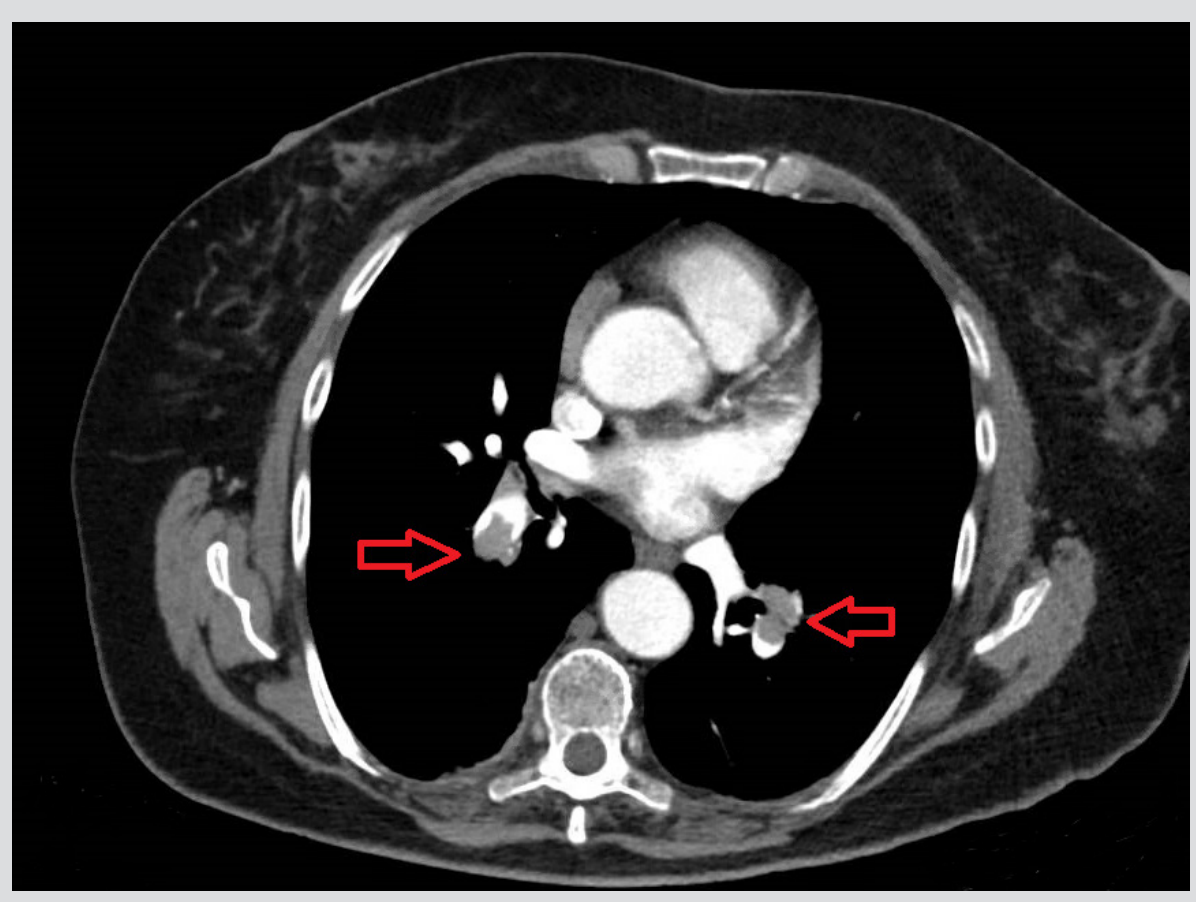

Figure 2. Bilateral pulmonary embolism (red arrows) demonstrated on chest CT angiography

\section{DISCUSSION}

High D-dimer blood levels might be features of both COVID-19 infection and PE. Previous reports focused on different thresholds of D-dimer blood levels upon admission to predict PE in COVID-19 patients ${ }^{[3-5]}$. We propose a different novel strategy to predict PE in COVID-19 patients. Both D-dimer and CRP blood levels are increased during the early stages of COVID-19 infection due to systemic inflammation ${ }^{[2]}$. However, over time one would expect D-dimer and CRP blood levels to gradually decrease together as the COVID-19 infection resolves. Hence, one should measure the blood levels of both biomarkers over time, and if there is a discrepancy with an increase in D-dimer blood levels and a decrease in CRP blood levels, then VTE and PE should be suspected since this phenomenon may represent resolution of the COVID-19 infection, but also ongoing and possibly worsening VTE in the absence of full-dose anticoagulation therapy. This theory should be studied in large cohorts, but it is already easy to implement clinically.

\section{REFERENCES}

1. Zhai Z, Li C, Chen Y, Gerotziafas G, Zhang Z, Wan J, et al. Prevention Treatment of VTE Associated with COVID-19 Infection Consensus Statement Group. Prevention and treatment of venous thromboembolism associated with coronavirus disease 2019 infection: a consensus statement before guidelines. Thromb Haemost 2020 Apr 21 . doi: 10.1055/s-0040-1710019 [Epub ahead of print].

2. Zhu J, Ji P, Pang J, Zhong Z, Li H, He C, et al. Clinical characteristics of 3,062 COVID-19 patients: a meta-analysis. J Med Virol 2020 Apr 15. doi: 10.1002/jmv.25884 [Epub ahead of print].

3. Huang C, Wang Y, Li X, Ren L, Zhao J, Hu Y, et al. Clinical features of patients infected with 2019 novel coronavirus in Wuhan, China. Lancet 2020;395:497-506.

4. Leonard-Lorant I, Delabranche X, Severac F, Helms J, Pauzet C, Collange O, et al. Acute pulmonary embolism in COVID-19 patients on CT angiography and relationship to D-dimer levels. Radiology 2020 Apr 23. doi: 10.1148/radiol.2020201561 [Epub ahead of print].

5. Cui S, Cui S, Chen S, Li X, Liu S, Wang F. Prevalence of venous thromboembolism in patients with severe novel coronavirus pneumonia. J Thromb Haemost 2020 Apr 9. doi: 10.1111/jth.14830 [Epub ahead of print]. 
European Journal

of Case Reports in

Internal Medicine 